\title{
Study on serum proteomic features in patients with and without recurrence or metastasis after surgical resection of esophageal carcinoma
}

G.B. Zheng, C.F. Gao, X.L. Wang, G. Zhao and D.H. Li

Institute of Anal-Colorectal Surgery, The 150th Central Hospital of Chinese PLA, Luoyang, Henan Province, China

Corresponding author: G.B. Zheng

E-mail: guobaozhengcn@126.com

Genet. Mol. Res. 13 (1): 538-545 (2014)

Received August 28, 2013

Accepted October 30, 2013

Published January 21, 2014

DOI http://dx.doi.org/10.4238/2014.January.21.23

\begin{abstract}
The purpose of this study was to identify specific biomarkers for recurrence or metastasis of esophageal carcinoma in serum of patients subjected to esophagectomy. Surface-enhanced laser desorption/ionization time-of-flight mass spectrometry (SELDI-TOF-MS) combined with IMAC- $\mathrm{Cu}^{2+}$ ProteinChip array were performed for the serum protein profiling in patients after surgical resection of esophageal carcinoma. Two groups of patients were analyzed: 38 patients without recurrence or metastasis (Group 1) and 22 patients with recurrence or metastasis after resection (Group 2). The Biomarker Wizard and Biomarker Patterns software were used to identify proteins differentially expressed between the 2 groups. There were 33 differentially expressed serum proteins detected by comparison between the groups. The classification tree model composed of 3 differentially expressed proteins with different $\mathrm{m} / \mathrm{z}(9368.63,5342.59$, and $5254.43 \mathrm{Da})$ was established. Under the learning mode, the sensitivity and specificity of this model for diagnosis of esophageal carcinoma recurrence or metastasis were
\end{abstract}


both $100 \%(22 / 22$ and $38 / 38$, respectively). Under the testing mode, the sensitivity and specificity were $90.9 \%(20 / 22)$ and $94.7 \%(36 / 38)$, respectively. The recurrence or metastasis of esophageal carcinoma after esophagectomy can be rapidly and accurately detected using the combination of SELDI-TOF-MS with IMAC-Cu ${ }^{2+}$ ProteinChip array, which, therefore, has a potential for clinical application.

Key words: Esophageal carcinoma; Radical operation; Recurrence; Metastasis; Biomarker

\section{INTRODUCTION}

Esophageal cancer is one of the most common malignancies of the gastrointestinal tract, and the sixth leading cause of cancer death worldwide (Blot and McLaughlin, 1999). The recurrence and metastasis after radical surgery is the main reason for poor prognosis and high mortality. Early detection of recurrent/metastatic cancer combined with positive complex therapy can result in a more effective treatment; however, at present, a convenient and rapid detection method is still lacking. Investigation of serum proteomic profiles to develop an effective diagnostic method is important for timely treatment of postoperative esophageal carcinoma recurrence and metastasis. Currently used diagnostic methods including gastroscopy, B ultrasound, computed tomography (CT), and magnetic resonance imaging have such disadvantages as traumatism, high cost, and complexity, and are therefore not quite suitable for early diagnosis of esophageal carcinoma recurrence and metastasis. In this study, surface enhanced laser desorption/ionization time-of-flight mass spectrometry (SELDI-TOF-MS) combined with IMAC- $\mathrm{Cu}^{2+}$ ProteinChip array was used to study the serum proteome in the esophageal carcinoma patients with and without recurrence or metastasis after esophagectomy for identification of cancer-specific serum biomarkers.

\section{MATERIAL AND METHODS}

\section{Research subjects and inclusion criteria}

Sixty patients (41 males and 19 females) with esophageal carcinoma admitted to the 150th Central Hospital of Chinese PLA from December 2006 to December 2007 were enrolled in this study. The participants were between 35 and 82 years of age, with an average of 58.7 years. All patients were diagnosed with esophageal squamous cell carcinoma by postoperative pathological examination, biopsy by gastroscopy, B-mode ultrasound, or CT-guided puncture. There were 38 cases without recurrence or metastasis after radical surgical resection of esophageal carcinoma (group 1) and 22 cases with recurrence or metastasis (group 2); blood collection after fasting was performed, respectively, in the morning of postoperative day 21 or the next morning after definite pathological diagnosis of recurrence or metastasis. In the group 1, the serum proteomic changes induced by factors related to surgery were excluded, and there were 3,14 , and 21 cases with stage I, II, and III cancer, respectively. In the group 2, there were 12, 4, and 6 cases with anastomotic recurrence, liver metastasis, and pulmonary metastasis, respectively. There was no significant difference in age, gender, or exposure history between the 2 groups. For all patients, the diseases that 
can induce production of serum interfering proteins (e.g., hepatitis, diabetes mellitus and connective tissue disease) were excluded. The consent was obtained from all patients or their families.

\section{Reagents and apparatus}

Cibacron blue, urea, acetonitrile, trifluoroacetic acid (TFA), and sinapinic acid (SPA) were purchased from Sigma Company, USA. SELDI-TOF-MS, IMAC-Cu ${ }^{2+}$ ProteinChip and assorted ProteinChip Biological System II-C (PBS II-C) were provided by CipherGen Company, USA.

\section{Serum sample collection}

Fasting blood $(5 \mathrm{~mL})$ was drawn from the cubital vein, placed in a refrigerator at $4{ }^{\circ} \mathrm{C}$ for $2 \mathrm{~h}$, and centrifuged $(700 \mathrm{~g}$ ) for $30 \mathrm{~min}$. The serum was transferred to 4 tubes $(100 \mu \mathrm{L}$ per tube), labeled, and preserved at $-70^{\circ} \mathrm{C}$. The same volume of fasting blood was drawn from a healthy person and subjected to the same treatment; the serum was transferred to 8 tubes, $100 \mu \mathrm{L}$ per tube.

\section{Serum sample pretreatment}

The serum samples were thawed and centrifuged $\left(4^{\circ} \mathrm{C}, 20,000 \mathrm{~g}\right)$ for $10 \mathrm{~min}$. For protein denaturation, $20 \mu \mathrm{L}$ sample was incubated with $40 \mu \mathrm{L}$ U9 buffer (containing $9 \mathrm{M}$ urea, $2 \%$ CHAPS, and $50 \mathrm{mM}$ Tris- $\mathrm{HCl}, \mathrm{pH}$ 9.0) for $30 \mathrm{~min}$ at room temperature with shaking. The degenerated serum sample of $10 \mu \mathrm{L}$ was added to $120 \mu \mathrm{L}$ IMAC buffer (containing $100 \mathrm{mM}$ sodium phosphate and $500 \mathrm{mM}$ sodium chloride, $\mathrm{pH} 7.0$,) in a 1.5-mL centrifuge tube and incubated at room temperature for $30 \mathrm{~min}$ with shaking. After dilution, a preliminary experimental sample was added to 8 pools in a IMAC $30-\mathrm{Cu}^{2+}$ protein chip. The reproducibility of mass spectrum among different chips and different pools in a chip was evaluated. The experimental samples were randomly divided into 8 groups, with 8 samples per group for groups 1 to 7 and 4 samples for the group 8 . The 8 samples of each group were added to 8 pools in a chip, respectively.

\section{Preparation of IMAC-C ${ }^{2+}$ protein chip}

Fifty microliters $100 \mathrm{mM} \mathrm{CuSO}_{4}$ solution was added to each chip pool. After shaking at room temperature for $5 \mathrm{~min}$, the residual $\mathrm{CuSO}_{4}$ solution was removed, followed by washing 5 times with deionized water and drying. Then, $50 \mu \mathrm{L} 100 \mathrm{mM}$ sodium acetate solution, $\mathrm{pH}$ 4.0, was added, followed by incubation at room temperature for $5 \mathrm{~min}$ with shaking; the residual sodium acetate solution was removed, followed by washing 5 times with deionized water and drying. Addition of $150 \mu \mathrm{L}$ IMAC buffer was followed by shaking for $5 \mathrm{~min}$, and residual liquid was removed; this step was repeated once. Then, $50 \mu \mathrm{L}$ diluted sample was added to each pool and incubated at room temperature for 60 min with shaking; the residual liquid was removed, followed by washing with $150 \mu \mathrm{L}$ IMAC buffer 3 times (each time, 5 min with shaking at room temperature); final rinse was performed with $1 \mathrm{mM}$ HEPES, pH 7.0. The chip was removed, and each pool was marked with a hydrophobic pen, followed by air drying. SPA $(2 \mathrm{mg})$ was mixed with $30 \mu \mathrm{L}$ acetonitrile and $30 \mu \mathrm{L}$ $1 \%$ TFA in a $1.5-\mathrm{mL}$ centrifugal tube and incubated at room temperature for $5 \mathrm{~min}$ with shaking to dissolve SPA, followed by centrifugation for $1 \mathrm{~min}$. Then, $0.5 \mu \mathrm{L}$ SPA solution was applied to each chip pool and air-dried, and then another $0.5 \mu \mathrm{L}$ SPA was added. 


\section{Detection of IMAC-C ${ }^{2+}$ protein chip}

NP20 chip with all-in-one standard protein was used for external mass calibration. Data were collected with MS settings of laser intensity, 175; detection sensitivity, 8; optimized molecular weight range, 1500-20,000; optimal focus center, 6000; data acquisition range, 2080; collection times, 100. The ProteinChip software (Ciphergen Company, USA) was used for reading and analyzing the data obtained with $\mathrm{IMAC}-\mathrm{Cu}^{2+}$ protein chip. The original data were collected using $1 \times 10^{9} \mathrm{~Hz} / \mathrm{s}$ speed, and the serum protein profiling was rapidly and accurately recorded. The mass-to-charge ratio $(\mathrm{m} / \mathrm{z})$ and relative protein content were marked on the abscissa and ordinate, respectively.

\section{Statistical analysis}

The Biomarker Wizard software was used for statistical analysis and calculation of the $\mathrm{P}$ value for the protein content with the same $\mathrm{m} / \mathrm{z}$ for different groups. The Biomarker Pattern software (using decision tree method to classify target objects) was used to group the training data, analyze the correlation, and construct the optimal classification tree model. The training samples were randomly processed, and the data were grouped in the detection model to verify the reliability of the established classification tree model.

\section{RESULTS}

\section{Analysis results by the BioMarkerWizard software}

The proteomic features in serum samples of the 2 patient groups were compared using the BioMarker Wizard software, and 33 differentially expressed proteins were identified $(\mathrm{P}<0.01)$ (Figure 1). The expression levels of 4 proteins $(7723.09,8093.55,5508.87$, and $7739.62 \mathrm{Da}$ ) were decreased, while the expression of the other 29 proteins was increased in the group 2 patients.

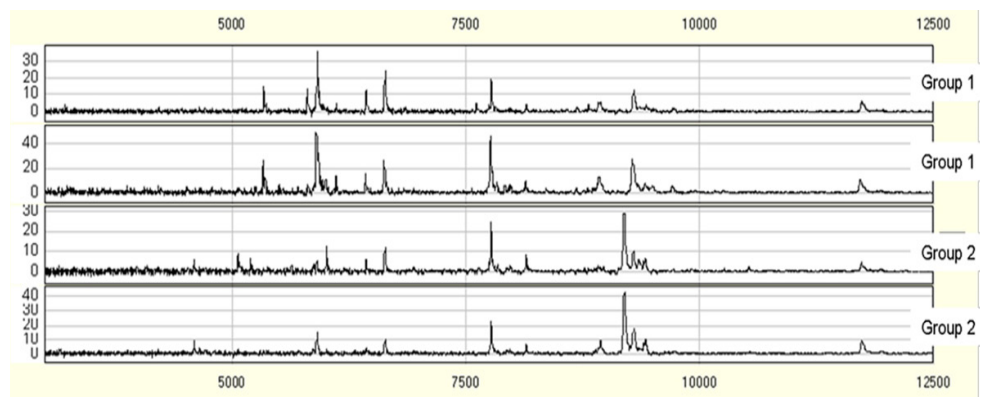

Figure 1. Serum protein profiling in 2 groups. The abscissa and ordinate present the $\mathrm{m} / \mathrm{z}$ and relative protein content, respectively.

\section{Analysis results by the Biomarker Pattern software}

If the content of protein with $\mathrm{m} / \mathrm{z}$ of $9368.63 \mathrm{Da}$ was not more than $4.520 \%$, the sample was separated from node 1 to node 2, otherwise to node 3 . If the content of protein 
with $\mathrm{m} / \mathrm{z}$ of $5254.42 \mathrm{Da}$ was not more than $4.383 \%$, the sample was separated from node 2 to terminal node 1 , otherwise to terminal node 2 . If the content of protein with $\mathrm{m} / \mathrm{z}$ of 5342.59 Da was not more than $10.240 \%$, the sample was separated from node 3 to terminal node 3 , otherwise to terminal node 4 . The serum samples separated to terminal nodes 1 and 3 belonged to the patients who did not experience recurrence or metastasis after radical surgical resection of esophageal carcinoma. The serum samples separated to terminal node 2 and 4 belonged to the patients who were diagnosed with cancer recurrence or metastasis after esophagectomy (Figure 2).

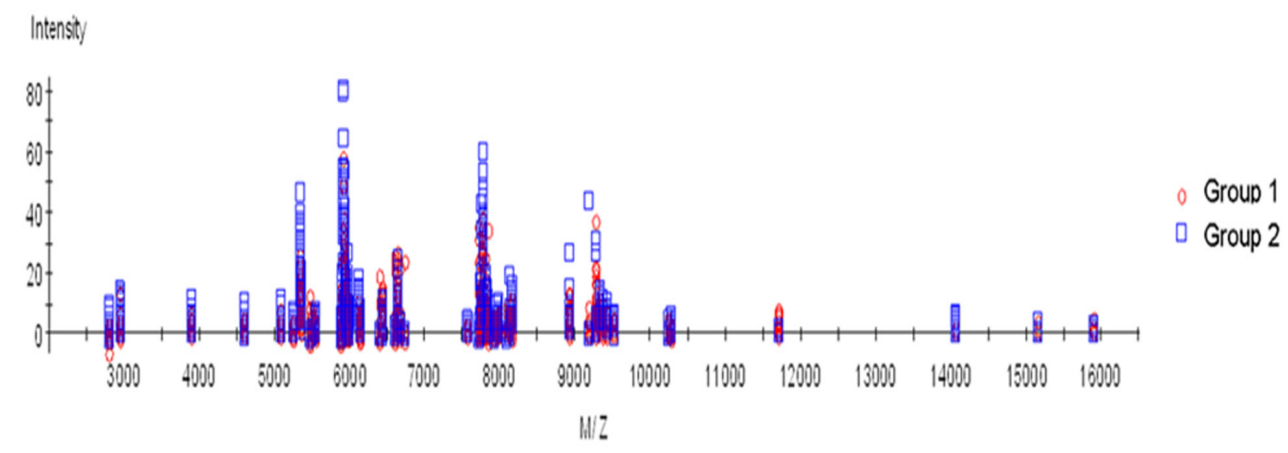

Figure 2. Proteins with differential expression in the serum of the group 1 and 2 patients.

The Biomarker Pattern software was used to analyze the proteins with differential expression in the group 1 and 2 patients; the results are shown in Figure 3. Three of the 33 differentially expressed proteins $(9368.63,5342.59$, and $5254.43 \mathrm{Da})$ were selected to build a classification tree model. Under the learning mode, the sensitivity and specificity of the classification tree model for prognosis of the esophageal carcinoma recurrence or metastasis after esophagectomy were both $100 \%$ (22/22 and $38 / 38$, respectively). The samples were randomly treated and grouped in the testing mode. Under this mode, the sensitivity and specificity of the classification tree model were $90.9 \%$ (20/22) and 94.7\% (36/38), respectively.

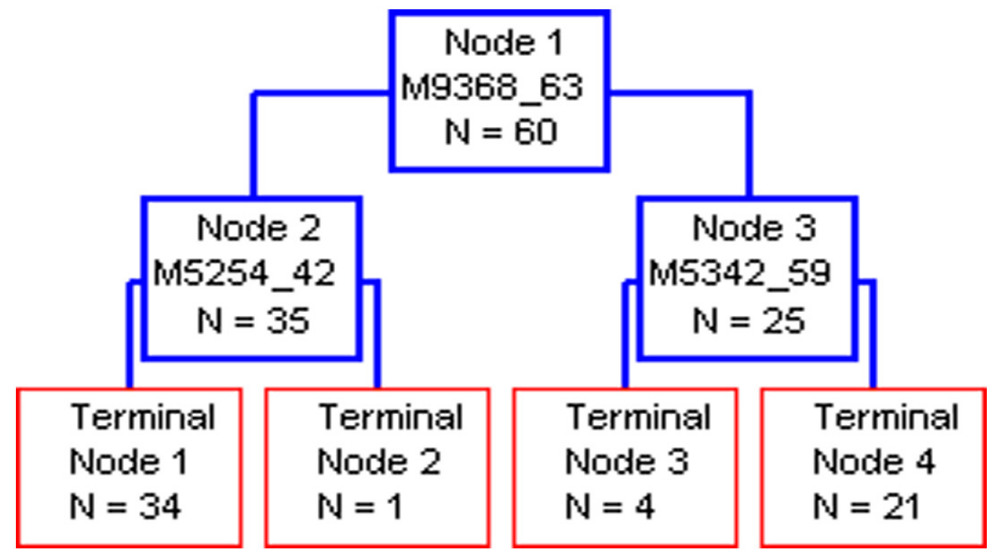

Figure 3. Classification tree model (learning mode). 


\section{DISCUSSION}

Esophageal carcinoma is a common malignancy with a high mortality. The effect of the combined chemotherapy and radiation therapy for patients with esophageal carcinoma is still not ideal. The 5-year survival rate for these patients is less than $30 \%$ because of the postoperative recurrence and metastasis. At present, the convenient and efficient detection method for early diagnosis of postoperative recurrence or metastasis is still lacking. The primary curative treatment for esophageal carcinoma is esophagectomy, but the second surgery is much more difficult, and the resection rates are low. For patients with early stages of esophageal carcinoma, the combination of chemotherapy and radiation therapy can be relatively effective, and for patients with early postoperative solitary metastatic lesion, the tumor resection or combined therapy can still produce good results. Therefore, early diagnosis of postoperative recurrence and metastasis is very important for improving the prognosis for patients with esophageal carcinoma. Existing diagnostic techniques and methods such as gastroscopy, exfoliative cytology test, and imaging examination have certain advantages in diagnosis of esophageal carcinoma recurrence or metastasis. However, accompanying trauma and pain and the high cost of these methods negatively affect patient compliance, and make these tests not quite suitable for large-scale screening and routine postoperative examination.

In recent years, development of serum biomarkers for esophageal carcinoma has become a research hotspot, and many promising candidates have been identified. For example, an increase in matrix metalloproteinase (MMP) level is considered an early indication of esophageal carcinoma metastasis and invasion (Koyama et al., 2000; Ohashi et al., 2000; Salmela et al., 2001; Yamashita et al., 2001; Ding et al., 2002; Mathew et al., 2002; Suzuki et al., 2002; Tanioka et al., 2003; Samantaray et al., 2004; El-Kenawy et al., 2005). Inducible nitric oxide synthase (iNOS) may be a high-risk marker for esophageal metaplasia, atypical hyperplasia, and malignancy (Jin et al., 2000), and interleukin 12 (IL-12) and interleukin 18 (IL-18) may be valuable diagnostic markers for esophageal carcinoma (Tsuboi et al., 2004). However, these markers are only in the initial stage of investigation, and their clinical diagnostic values still need to be further confirmed. Application of these markers for diagnosis of postoperative recurrence and metastasis of esophageal carcinoma has not been reported. Since the beginning of this century, SELDI-TOF-MS has been used to determine tumor-associated proteins (Jr et al., 1999; Adam et al., 2001; Paweleta et al., 2001; Vlahou et al., 2001; Chapman, 2002; Issaq et al., 2002; Li et al., 2002; Wiesner, 2004; Yang et al., 2005; Pan et al., 2006; $\mathrm{Xu}$ et al., 2006). This technique provides a high-end technology platform for rapid, accurate, and high-throughput screening of specific tumor biomarkers in the body fluids. In this study, SELDI-TOF-MS combined with IMAC-Cu ${ }^{2+}$ ProteinChip array was used to identify specific biomarkers in serum of patients diagnosed with esophageal carcinoma recurrence or metastasis. The objective was to develop a convenient and rapid detection method for early diagnostics of patients with recurrence of esophageal carcinoma, which will lead to improvement in the complex treatment outcomes.

In this study, the classification tree model composed of 3 differential proteins with different $\mathrm{m} / \mathrm{z}(9368.63,5342.59$, and $5254.43 \mathrm{Da})$ is constructed. The sensitivity and specificity of this model for the recurrence and metastasis of esophageal carcinoma after radical operation under the learning mode are both $100 \%$ (22/22 and 38/38, respectively), and under the testing mode, they are $90.9 \%(20 / 22)$ and $94.7 \%(36 / 38)$, respectively. It is believed that this 
diagnostic model has better monitoring capacity with regard to early diagnosis of postoperative esophageal carcinoma recurrence and metastasis and promising potential for application in clinical setting.

Results of this study of serum proteins in the 2 patient groups show that the expression levels of 29 of the 33 differentially expressed proteins in group 2 were significantly increased, which may be due to the proteins or peptides secreted by tumor cells or induced by immune response to tumor growth and proliferation. The expression levels of the other 4 differentially expressed proteins were decreased. The exact cause of this effect is unknown, but the recently proposed immunoediting theory $(\mathrm{Cao}, 2007)$ suggests that a variety of tumor-secreted factors such as IL-10 and TGF- $\beta$ can have strong inhibitory effect on the immune system. For example, TGF- $\beta$ can inhibit signal transduction pathway initiated by cytokines, resulting in blocking of the perforation protein synthesis and inhibition of the immunoglobulin secretion, and the inhibitory effect can increase with the tumor growth. This may be one of the reasons for the reduced expression of some serum proteins in patients with postoperative esophageal carcinoma recurrence and metastasis.

The sample size in this study is small. Further investigations are needed to confirm if the established classification tree model can be finally used for prognosis of the postoperative esophageal carcinoma recurrence and metastasis. Next, the follow up for patients with radical surgical resection of esophageal carcinoma should be conducted to obtain paired serological samples for further evaluation of the specific biomarker potential for early disease detection.

The recurrence and metastasis of esophageal carcinoma after esophagectomy can be rapidly and accurately detected using combination of SELDI-TOF-MS with IMAC-Cu ${ }^{2+}$ ProteinChip array, which has a potential for application in clinical settings.

\section{ACKNOWLEDGMENTS}

Research supported by Medical Research Fund of Chinese PLA (\#08Z006).

\section{REFERENCES}

Adam BL, Vlahou A, Semmes OJ and Wright GL, Jr. (2001). Proteomic approaches to biomarker discovery in prostate and bladder cancers. Proteomics 1: 1264-1270.

Blot WJ and McLaughlin JK (1999). The changing epidemiology of esophageal cancer. Semin. Oncol. 26: 2-8.

Cao XT (2007). Growth and metastasis of tumors: Forum on immunological perspective. Chin. J. Cancer Biother. 14: 2-6.

Chapman K (2002). The ProteinChip Biomarker System from Ciphergen Biosystems: a novel proteomics platform for rapid biomarker discovery and validation. Biochem. Soc. Trans. 30: 82-87.

Ding Y, Shimada Y, Gorrin-Rivas MJ, Itami A, et al. (2002). Clinicopathological significance of human macrophage metalloelastase expression in esophageal squamous cell carcinoma. Oncology 63: 378-384.

El-Kenawy A, Lotfy M, El-Kott A and El-Shahat M (2005). Significance of matrix metalloproteinase 9 and CD34 expressions in esophageal carcinoma: correlation with DNA content. J. Clin. Gastroenterol. 39: 791-794.

Issaq HJ, Veenstra TD, Conrads TP and Felschow D (2002). The SELDI-TOF MS approach to proteomics: protein profiling and biomarker identification. Biochem. Biophys. Res. Commun. 292: 587-592.

Jin Y, Zhang W, Liu B, Wang H, et al. (2000). Expression of inducible nitric oxide synthase in human esophageal biopsies from carcinoma and precancerous lesions. Zhongguo Yi Xue Ke. Xue Yuan Xue Bao 22: 570-572.

Jr GW, Cazares LH, Leung SM, Nasim S, et al. (1999). Proteinchip(R) surface enhanced laser desorption/ionization (SELDI) mass spectrometry: a novel protein biochip technology for detection of prostate cancer biomarkers in complex protein mixtures. Prostate Cancer Prostatic. Dis. 2: 264-276.

Koyama H, Iwata H, Kuwabara Y, Iwase H, et al. (2000). Gelatinolytic activity of matrix metalloproteinase-2 and -9 in oesophageal carcinoma; a study using in situ zymography. Eur. J. Cancer 36: 2164-2170. 
Li J, Zhang Z, Rosenzweig J, Wang YY, et al. (2002). Proteomics and bioinformatics approaches for identification of serum biomarkers to detect breast cancer. Clin. Chem. 48: 1296-1304.

Mathew R, Khanna R, Kumar R, Mathur M, et al. (2002). Stromelysin-2 overexpression in human esophageal squamous cell carcinoma: potential clinical implications. Cancer Detect. Prev. 26: 222-228.

Ohashi K, Nemoto T, Nakamura K and Nemori R (2000). Increased expression of matrix metalloproteinase 7 and 9 and membrane type 1-matrix metalloproteinase in esophageal squamous cell carcinomas. Cancer 88: 2201-2209.

Pan YZ, Xiao XY, Zhao D, Zhang L, et al. (2006). Application of surface-enhanced laser desorption/ionization time-offlight-based serum proteomic array technique for the early diagnosis of prostate cancer. Asian J. Androl. 8: 45-51.

Paweleta CP, Trock B, Pennanen M and Tsangaris T (2001). Proteomic patterus of nipple aspirate fluids obtained by SELDI-TOF : potential for new biomarkersto aid in the diagnosis of breast cancer. DisMarkers 17: 301-307.

Salmela MT, Karjalainen-Lindsberg ML, Puolakkainen P and Saarialho-Kere U (2001). Upregulation and differential expression of matrilysin (MMP-7) and metalloelastase (MMP-12) and their inhibitors TIMP-1 and TIMP-3 in Barrett's oesophageal adenocarcinoma. Br. J. Cancer 85: 383-392.

Samantaray S, Sharma R, Chattopadhyaya TK, Gupta SD, et al. (2004). Increased expression of MMP-2 and MMP-9 in esophageal squamous cell carcinoma. J. Cancer Res. Clin. Oncol. 130: 37-44.

Suzuki T, Kuwabara Y, Iwata H, Mitani M, et al. (2002). Role of matrix metalloproteinase-9 in in vitro invasion of esophageal carcinoma cells. J. Surg. Oncol. 81: 80-86.

Tanioka Y, Yoshida T, Yagawa T, Saiki Y, et al. (2003). Matrix metalloproteinase-7 and matrix metalloproteinase-9 are associated with unfavourable prognosis in superficial oesophageal cancer. Br. J. Cancer 89: 2116-2121.

Tsuboi K, Miyazaki T, Nakajima M, Fukai Y, et al. (2004). Serum interleukin-12 and interleukin-18 levels as a tumor marker in patients with esophageal carcinoma. Cancer Lett. 205: 207-214.

Vlahou A, Schellhammer PF, Mendrinos S, Patel K, et al. (2001). Development of a novel proteomic approach for the detection of transitional cell carcinoma of the bladder in urine. Am. J. Pathol. 158: 1491-1502.

Wiesner A (2004). Detection of tumor markers with ProteinChip technology. Curr. Pharm. Biotechnol. 5: 45-67.

$\mathrm{Xu}$ WH, Chen YD, Hu Y, Yu JK, et al. (2006). Preoperatively molecular staging with CM10 ProteinChip and SELDITOF-MS for colorectal cancer patients. J. Zhejiang Univ. Sci. B 7: 235-240.

Yamashita K, Mori M, Kataoka A, Inoue H, et al. (2001). The clinical significance of MMP-1 expression in oesophageal carcinoma. Br. J. Cancer 84: 276-282.

Yang SY, Xiao XY, Zhang WG, Zhang LJ, et al. (2005). Application of serum SELDI proteomic patterns in diagnosis of lung cancer. BMC Cancer 5: 83. 